\title{
ON CERTAIN TWO-POINT PROPERTIES OF GENERAL FAMILIES
}

\author{
OF CURVES*
}

\author{
BY \\ JESSE DOUGLAS
}

In a paper published in $1903, \dagger R$. Straubel proved an important theorem of geometrical optics to the following effect. In any isotropic medium,-in which the index of refraction is therefore a function of position only, $\nu(x, y$, $z$ ), - let $a$ and $b$ be any two points. Imagine an element of area $\delta \sigma_{1}$ to be placed at $b$ normal to the (curvilinear) ray $a b$, and let it subtend at $a$ the solid angle $\delta \omega_{1}$. Reversing the situation, suppose an element $\delta \sigma_{2}$ placed likewise at $a$ to subtend at $b$ the solid angle $\delta \omega_{2}$. Then the relation

$$
\frac{\delta \sigma_{1}}{\delta \omega_{1}}: \nu^{2}(a)=\frac{\delta \sigma_{2}}{\delta \omega_{2}}: \nu^{2}(b)
$$

will subsist. In $n$ dimensions $\nu^{n-1}$ takes the place of $\nu^{2}$.

Straubel's theorem was made the subject of a paper by T. Levi-Civita, $\ddagger$ who generalized it to the case of a non-isotropic medium. Levi-Civita's fundamental result is as follows. Consider the geodesics of a general (curved) $n$-dimensional variety $V_{n}$. Denoting by $a$ and $b$ any two points in $V_{n}$, let an $(n-1)$-dimensional element of extent $\delta \sigma_{1}$ placed at $b$ normal to the geodesic $a b$ subtend at $a$ the solid angle $\delta \omega_{1}$. If then an element $\delta \sigma_{2}$ placed likewise at $a$ subtends at $b$ the solid angle $\delta \omega_{2}$, we shall have

$$
\frac{\delta \sigma_{1}}{\delta \omega_{1}}=\frac{\delta \sigma_{2}}{\delta \omega_{2}}
$$

The object of the present paper is to develop general geometric theorems in the above order of ideas for an arbitrary family of curves. In particular, the property of Levi-Civita's theorem is shown to hold for a wider class

* Presented to the Society, April, 1917. See Bulletin of the American Mathematical Societ y, vol. 23 (1916-17), p. 441.

$\dagger$ Ueber einen allgemeinen Satz der geometrischen Optik und einige Anwendungen, $\mathrm{P}$ h y $8 \mathrm{i}-$ kalis che Z eit s chrift, vol. 4 (1903), pp. 114-117.

† Una proprieta de simmetria delle traiettorie dinamiche spiccati da due punti, A t t i d e 11 a Reale Accademia dei Lincei, vol. 24, April, 1915. The notation is different from that of the present paper. 
of curve families than the geodesics. Straubel's theorem is discussed as to its purely geometrical implications; it is shown, among other things, that the Straubel relation is not characteristic of the curve families that can arise as the totality of paths of light in an isotropic medium,-that is, of the natural families, so called.*

The method of investigation is characterized by the systematic use of the fact that, given a family of curves defined by any system of differential equations, the curves infinitesimally near to a given one obey a system of linear differential equations - the equations of variation (Poincaré) or auxiliary equations (Darboux) of those that define the given curve family.

\section{Theory for two dimensions}

1. Definitions. The functions $V(a, b)$ and $W(a, b)$. Consider any family of $\infty^{2}$ curves in the plane, as defined by a differential equation of the second order, $y^{\prime \prime}=F\left(x, y, y^{\prime}\right)$.

Let $a$ and $b$ be any two points of the plane. Then, under suitable restrictions, $\dagger$ there is a unique curve $C$ of the given family joining $a$ and $b$. Imagine an infinitesimal element $b b^{\prime}$ of length $\delta \sigma_{\mathrm{t}}$ to be placed at $b$ normal to $C$, and let $C_{1}$ be the curve of the given family that joins $b^{\prime}$ to $a$. Denoting by $\delta \omega_{1}$ the infinitesimal angle enclosed at $a$ between $C_{1}$ and $C$, the ratio $\delta \sigma_{1} / \delta \omega_{1}$ is a function of the two points $a$ and $b$; we call it $V(a, b)$.

Similarly, let the element $a a^{\prime}$ of length $\delta \sigma_{2}$ be normal to $C$ at $a$, and let $C_{2}$ denote the curve that passes through $a^{\prime}$ and $b$. Then designating as $\delta \omega_{2}$ the angle at $b$ between $C_{2}$ and $C$, the ratio $\delta \sigma_{2} / \delta \omega_{2}$ is appropriately $V(b, a)$. It is to be remarked that in general $V(a, b) \neq V(b, a)$.

Besides the defining equations

$$
V(a, b)=\frac{\delta \sigma_{1}}{\delta \omega_{1}}, \quad V(b, a)=\frac{\delta \sigma_{2}}{\delta \omega_{2}},
$$

we introduce also the notation

$$
W(a, b)=\frac{V(a, b)}{V(b, a)} .
$$

Our specific object is the study of the geometric functions $V(a, b), W(a, b)$.

2. Expression for $V(a, b)$. We have under consideration a doubly-infinite system of curves, defined by a differential equation of the second order,

$$
Y^{\prime \prime}=F\left(X, Y, Y^{\prime}\right) \text {. }
$$

To every set of initial values $x, y, p$ for $X, Y, Y^{\prime}$ there corresponds a

* After E. Kasner, Differential-geometric aspects of dynamics, Princet on Collo quium Lectures (1912), pp. 34-72, or his papers in these Transactions, vol. 10 (1909), p. 72, and vol. 11 (1910); p. 121, where several sets of completely characteristic properties of natural families are given.

† See Picard, Traité d'Analyse, vol. 3, pp. 90-99. 
definite curve; let its equation be

$$
Y=Y(X ; x, y, p) \text {. }
$$

Explicitly, we may write $Y$ as a power series; the first terms are

$$
Y=y+p(X-x)+\frac{1}{2} F(x, y, p)(X-x)^{2}+\cdots
$$

Let the coördinates of $a$ be $\left(x_{1}, y_{1}\right)$, those of $b,\left(x_{2}, y_{2}\right)$; let the slope of the curve $C$ be $p_{1}$ at $a$, and $p_{2}$ at $b$. Then in (2), $C$ corresponds to the initial values $x_{1}, y_{1}, p_{1}$; also to the initial values $x_{2}, y_{2}, p_{2}$. Suppose that for these values $(2)$ reduces to

$$
Y=y(X),
$$

which is then the equation of $C$.

The curve $C_{1}$ will correspond to

$$
x=x_{1}, \quad y=y_{1}, \quad p=p_{1}+\delta p_{1},
$$

denoting by the last the slope of $C_{1}$ at $a$; the equation of $C_{1}$ will be therefore

$$
Y=y(X)+\delta p_{1} Y_{p}\left(X ; x_{1}, y_{1}, p_{1}\right),
$$

where the powers of $\delta p_{1}$ beyond the first have been neglected as infinitesimals of higher order. The subscript $p$ denotes partial differentiation with respect to that variable.

Since $x_{1}, y_{1}, p_{1}$ are fixed, $Y_{p}$ is a function of $X$ alone; we introduce for it the notation

$$
\eta_{1}(X)=Y_{p}\left(X ; x_{1}, y_{1}, p_{1}\right),
$$

and write the equation of $C_{1}$ in the form

$$
Y=y(X)+\delta p_{1} \eta_{1}(X) \text {. }
$$

In the same way, the equation of $C_{2}$ will be

$$
Y=y(X)+\delta p_{2} \eta_{2}(X)
$$

where

$$
\eta_{2}(X)=Y_{p}\left(X ; x_{2}, y_{2}, p_{2}\right) \text {. }
$$

In the figure of the preceding section let $b b^{\prime \prime}$ denote the element of length at $b$ parallel to the $y$-axis and included between the curves $C$ and $C_{1}$. Obviously,

Hence

$$
b b^{\prime \prime}=\delta p_{1} \eta_{1}\left(x_{2}\right)
$$

Also

$$
\delta \sigma_{1}=b b^{\prime \prime} \cos \varangle b^{\prime} b b^{\prime \prime}=\frac{\delta p_{1} \eta_{1}\left(x_{2}\right)}{\left(1+p_{2}^{2}\right)^{1 / 2}} .
$$

$$
\delta \omega_{1}=\delta \tan ^{-1} p_{1}=\frac{\delta p_{1}}{1+p_{1}^{2}}
$$


Therefore

$$
V(a, b)=\frac{\delta \sigma_{1}}{\delta \omega_{1}}=\frac{1+p_{1}^{2}}{\left(1+p_{2}^{2}\right)^{1 / 2}} \eta_{1}\left(x_{2}\right) .
$$

To obtain the formula for $V(b, a)$, we have only to interchange systematically the rôles of $a$ and $b$. The result is

$$
V(b, a)=\frac{\delta \sigma_{2}}{\delta \omega_{2}}=-\frac{1+p_{2}^{2}}{\left(1+p_{1}^{2}\right)^{1 / 2}} \eta_{2}\left(x_{1}\right),
$$

where the significance of the minus sign is as follows: in forming $V(a, b)$ the curve $C$ is viewed in the sense $a b$; in forming $V(b, a)$, in the reverse sense $b a$.

3. Expression for $W(a, b)$. The important fact concerning $\eta_{1}(X)$ and $\eta_{2}(X)$ is that they are two independent solutions of a certain linear differential equation of the second order.* To see this, return to the equations (1) and (2) of the preceding section. (2) represents the general solution of (1); this means that we have identically in the four variables $X, x, y, p$ :

$$
Y^{\prime \prime}(X ; x, y, p)=F\left[X, Y(X ; x, y, p), Y^{\prime}(X ; x, y, p)\right],
$$

where the accents denote partial differentiation with respect to $X$. We may differentiate this identity with respect to $p$, obtaining:

$$
\left(Y^{\prime \prime}\right)_{p}=F_{y}\left(Y_{p}\right)+F_{y^{\prime}}\left(Y^{\prime}\right)_{p} \cdot \dagger
$$

Under the assumption that all the derivatives involved are continuous, the differentiations with respect to $X$ and $p$ are commutative; the above equation may therefore be rewritten:

$$
\left(Y_{p}\right)^{\prime \prime}=F_{y}\left(Y_{p}\right)+F_{y^{\prime}}\left(Y_{p}\right)^{\prime} .
$$

When $x, y, p$ are set equal respectively to $x_{1}, y_{1}, p_{1}, Y_{p}$ reduces to $\eta_{1}(X)$; when the same letters are equal to $x_{2}, y_{2}, p_{2}, Y_{p}$ reduces to $\eta_{2}(X)$. It follows that $\eta_{1}(X)$ and $\eta_{2}(X)$ obey the differential equation

$$
\eta^{\prime \prime}(X)=F_{y^{\prime}} \eta^{\prime}(X)+F_{y} \eta(X),
$$

where it is understood that in $F_{y^{\prime}}\left(X, Y, Y^{\prime}\right), F_{y}\left(X, Y, Y^{\prime}\right)$ we are to substitute for $Y$ and $Y^{\prime}$ from the curve $C$ :

$$
Y=y(X), \quad Y^{\prime}=y^{\prime}(X),
$$

so that these coefficients are functions of $X$ alone, where $X$ represents the abscissa along $C$.

The functions $\eta_{1}, \eta_{2}$ will be completely defined by giving their values and those of their first derivatives for an initial value of $X$. These are determined as follows. Differentiating $\left(2^{\prime}\right)$ with respect to $p$, we have, after writing $x$;

* The equation of variation of the differential equation (1).

t For convenience of notation, small letters are used instead of capitals in denoting the partial derivatives of the function $F$. 
$y, p$ equal to $x_{1}, y_{1}, p_{1}$ :

hence

$$
\eta_{1}(X)=\left(X-x_{1}\right)+\frac{1}{2} F_{p}\left(x_{1}, y_{1}, p_{1}\right)\left(X-x_{1}\right)^{2}+\cdots,
$$

Similarly we find

$$
\eta_{1}\left(x_{1}\right)=0, \quad \eta_{1}^{\prime}\left(x_{1}\right)=1
$$

$$
\eta_{2}\left(x_{2}\right)=0, \quad \eta_{2}^{\prime}\left(x_{2}\right)=1 \text {. }
$$

Now $\eta_{1}(X), \eta_{2}(X)$, as two independent solutions of the linear differential equation (6), must obey the relation

$$
\left|\begin{array}{ll}
\eta_{1}(X) & \eta_{2}(X) \\
\eta_{1}^{\prime}(X) & \eta_{2}^{\prime}(X)
\end{array}\right|=e^{\int_{k}^{x} F_{y^{\prime}} d X},
$$

where $k$ is an undetermined constant.

In this write successively

$$
X=x_{1}, \quad X=x_{2},
$$

and take account of the equations $\left(7_{1}\right),\left(7_{2}\right)$; there results:

$$
\begin{aligned}
-\eta_{2}\left(x_{1}\right) & =e^{\int_{k}^{x_{1}} F_{y^{\prime}} d x}, \\
\eta_{1}\left(x_{2}\right) & =e^{\int_{k}^{x_{2}} F_{y^{\prime}} d x} .
\end{aligned}
$$

Dividing the second equation by the first, the unknown $k$ is eliminated:

$$
-\frac{\eta_{1}\left(x_{2}\right)}{\eta_{2}\left(x_{1}\right)}=e^{\int_{x_{1}}^{x_{x}} F_{y^{\prime}} d x} \text {. }
$$

The result thus obtained applies directly to the derivation of an expression for $W(a, b)$. By definition,

$$
W(a, b)=\frac{V(a, b)}{V(b, a)}
$$

so that, substituting the expressions $\left(5_{1}\right),\left(5_{2}\right)$ for $V(a, b), V(b, a)$, we have

Hence, by equation (8),

$$
W(a, b)=-\frac{\left(1+p_{1}^{2}\right)^{3 / 2} \cdot \frac{\eta_{1}\left(x_{2}\right)}{\left(1+p_{2}^{2}\right)^{3 / 2}}}{\eta_{2}\left(x_{1}\right)} .
$$

$$
W(a, b)=\frac{\left(1+p_{1}^{2}\right)^{3 / 2}}{\left(1+p_{2}^{2}\right)^{3 / 2}} e^{x_{x_{1}}^{x_{2}} F_{y^{\prime}} d x} .
$$

This can easily be transformed into

$$
W(a, b)=e^{\int_{x_{1}}^{x_{2}}\left(F_{y^{\prime}}-\frac{3 y^{\prime} F}{1+y^{\prime 2}}\right) d x} .
$$

The function $W(a, b)$ is an intrinsic geometric quantity, being, that is to say, independent of the selection of coördinate axes. It is desirable to find for it an expression in which only intrinsic elements shall figure. 
This is attained through the following considerations. We are dealing with a family of $\infty^{2}$ curves of which one passes through every point in every direction. Let $\partial \gamma / \partial \theta$ denote the rate of change of curvature with respect to variation of direction as, remaining at any fixed point, we pass from curve to curve of the family. The expression for $\partial \gamma / \partial \theta$ is

$$
\frac{\partial \gamma}{\partial \theta}=\frac{\left(1+y^{\prime 2}\right) F_{y^{\prime}}-3 y^{\prime} F}{\left(1+y^{\prime 2}\right)^{3 / 2}} ;
$$

and to pass from this to the integrand of (9), we have only to introduce the element of arc along $\dot{C}$,

Multiplying, we get

$$
d s=\left(1+{y^{\prime 2}}^{1 / 2} d x .\right.
$$

$$
\frac{\partial \gamma}{\partial \theta} d s=\left(F_{y^{\prime}}-\frac{3 y^{\prime} F}{1+y^{\prime 2}}\right) d x ;
$$

therefore, according to (9),

$$
W(a, b)=e^{\int_{a}^{b} \frac{\partial \gamma}{\partial \theta} d s} .
$$

The path of integration is the arc $a b$ of the curve $C$.

4. The multiplicative property of $W(a, b)$. From the result (10) certain simple corollaries follow directly.

Consider any three points $a, b, c$ located not in general position but on the same curve $C$ of the double infinite family under consideration. We have

$$
W(a, b)=e^{\int_{a}^{b} \frac{\partial \gamma}{\partial \theta} d s}, \quad W(b, c)=e^{\int_{b}^{c} \frac{\partial \gamma}{\partial \theta} d s}, \quad W(a, c)=e^{\int_{a}^{c} \frac{\partial \gamma}{\partial \theta} d s} .
$$

Multiplying together the first two of these equations and taiking account of the additive nature of integration, we find

$$
W(a, b) \cdot W(b, c)=W(a, e) .
$$

Along any individual curve $C$ the function $W(a, b)$ is of multiplicative character.

5. Transitivity of the relation $V(a, b)=V(b, a)$. A second result follows as a sub-corollary of (11). We have remarked that in general $V(a, b)$ $\neq V(b, a)$. But suppose, as a special situation, that $a, b, c$ are three points $n n$ the same curve $C$, and that

$$
V(a, b)=V(b, a), \quad \text { and } \quad V(b, c)=V(c, b) .
$$

Then it must follow as a consequence that

$$
V(a, c)=V(c, a) ;
$$

for the above relations are equivalent to

$$
W(a, b)=1, \quad W(b, c)=1,
$$


from which by (11) it follows that

or that

$$
W(a, c)=1,
$$

$$
V(a, c)=V(c, a) .
$$

Thus, along any individual curve $C$, the relation $V(a, b)=V(b, a)$ is a transitive one. We remark that this fact is not at all evident from the geometric definition of $V(a, b)$.

6. Curve families with symmetric $V(a, b)$. The particular doubly infinite systems of curves for which $V(a, b)$ is a symmetric function of the two points $a$ and $b$ are found immediately from a consideration of (10). We wish to have

which is equivalent to

$$
\begin{gathered}
V(a, b) \equiv V(b, a), \\
W(a, b) \equiv 1,
\end{gathered}
$$

or again, equivalent to

$$
\int_{a}^{b} \frac{\partial \gamma}{\partial \theta} d s=0
$$

for any two points $a$ and $b$. For this, the integrand $\partial \gamma / \partial \theta$ must be identically zero. Recalling the meaning of $\partial \gamma / \partial \theta$, we have therefore the

THEOREM. The necessary and sufficient condition that, for a family of $\infty^{2}$ curves in the plane, the corresponding two-point function, $V(a, b), b e$ symmetric, is that at any given point the curvature be the same for all the curves of the family through that point.

This means that $\gamma$ may be a function of $x, y$, but not of $y^{\prime}$. Curve families with symmetric $V(a, b)$ are therefore characterized by differential equations of the form

$$
y^{\prime \prime}=\left(1+{y^{\prime 2}}^{2 / 2} G(x, y) .\right.
$$

7. Straubel's theorem. Geometric interpretation. The theorem of Straubel stated at the beginning of this paper follows from applying the formula for $W(a, b)$ to the case of a family of light rays.

Imagine in the plane an isotropic optical medium, in which the index of refraction is $\nu(x, y)$. Such a medium gives rise to a system of $\infty^{2}$ curves as paths of light, these being determined as the extremals of the calculus of variations problem

$$
\int \nu(x, y) d s=\min .,
$$

or by the equivalent Euler-Lagrange differential equations:

$$
y^{\prime \prime}=\left(L_{y}-y^{\prime} L_{x}\right)\left(1+y^{\prime 2}\right)
$$

where $L=\log \nu$. Following $\mathrm{E}$. Kasner, ${ }^{*}$ we shall term a family of curves of the type (13) a natural family.

\footnotetext{
*Princeton Colloquium Lectures, pp. 34-37.
} 
In applying the formula (9) to natural families, we have

therefore

$$
F_{y^{\prime}}-\frac{3 y^{\prime} F}{1+y^{\prime 2}}=-L_{x}-y^{\prime} L_{y}=-\frac{d}{d x} L(x . y),
$$

that is

$$
W(a, b)=\frac{e^{L\left(x_{1}, y_{1}\right)}}{e^{L\left(x_{2}, y_{2}\right)}}=\frac{\nu\left(x_{1}, y_{1}\right)}{\nu\left(x_{2}, y_{2}\right)},
$$

which is Straubel's result.

$$
\frac{V(a, b)}{V(b, a)}=\frac{\nu(a)}{\nu(b)}
$$

Straubel's theorem is not purely geometric in so far as it contains the indices of refraction $\nu(a), \nu(b)$. An exclusively geometric form is obtainable by considering any three points $a, b, c$ of the plane. We shall have

$$
W(a, b)=\frac{\nu(a)}{\nu(b)}, \quad W(b, c)=\frac{\nu(b)}{\nu(c)}, \quad W(a, c)=\frac{\nu(a)}{\nu(c)},
$$

from which equations the indices of refraction can be eliminated, giving

Therefore:

$$
W(a, b) \cdot W(b, c)=W(a, c) .
$$

The multiplicative relation (11) which holds in the case of any doubly infinite family of curves when $a, b, c$ are on the same curve, holds in the case of a natural family for general position of $a, b, c$.

This is the purely geometric content of Straubel's theorem.

8. Converse of Straubel's theorem. Is the Straubel property characteristic of natural families? For the answer we return to (14). When can we define a function $\nu(x, y)$ of the points of the plane and have for any two points $a$ and $b$ :

$$
\frac{V(a, b)}{V(b, a)}=\frac{\nu(a)}{\nu(b)} ?
$$

By (9), the condition in question is equivalent to

$$
\int_{x_{1}}^{x_{2}}\left(F_{y^{\prime}}-\frac{3 y^{\prime} F}{1+y^{\prime 2}}\right) d x=\log \nu\left(x_{1}, y_{1}\right)-\log \nu\left(x_{2}, y_{2}\right)=-\left.L(x, y)\right|_{x_{1}, y_{1}} ^{x_{2}, y_{2}} \text {. }
$$

For this relation the necessary and sufficient condition is

$$
F_{y^{\prime}}-\frac{3 y^{\prime} F}{1+y^{\prime 2}}=-\frac{d}{d x} L(x, y)=-L_{x}-y^{\prime} L_{y} \text {. }
$$

The last is to be regarded as a partial differential equation for $F$. It can be integrated by means of the integrating factor $1 /\left(1+y^{\prime 2}\right)^{\prime}$, using which, we find

$$
\frac{F}{\left(1+y^{\prime 2}\right)^{\prime}}=\frac{L_{y}-y^{\prime} L_{x}}{\left(1+y^{\prime 2}\right)^{1}}+G(x, y)
$$


where $G(x, y)$ is the constant of integration (with respect to $y^{\prime}$ ). Thus we obtain for $F$ the expression

$$
F=\left(L_{y}-y^{\prime} L_{x}\right)\left(1+y^{\prime 2}\right)+G(x, y)\left(1+y^{\prime 2}\right)^{\prime},
$$

and have the theorem:

The Straubel property is not characteristic of natural families-it holds for the more general type defined by (15).*

9. Theory for an arbitrary surface. In extending the theory of the functions $V$ and $W$ to a family of $\infty^{2}$ curves upon a general surface, no new difficulties present themselves apart from the increased length of calculation. The typical feature is that the geodesic curvature $\gamma_{0}$ replaces the $\gamma$ of the plane.

The main result arrived at is the formula for $W(a, b)$ :

$$
W(a, b)=e^{\int_{a}^{b} \frac{\partial \gamma_{0}}{\partial \theta} d s}
$$

We consider particularly the special systems of $\infty^{2}$ curves for which $V(a, b)$ $\equiv V(b, a)$. Evidently, these are the ones for which $\partial \gamma_{o} / \partial \theta \equiv 0$, hence the

Theorem. The necessary and sufficient condition that a family of $\infty^{2}$ curves upon an arbitrary surface have the property $V(a, b) \equiv V(b, a)$ is that the geodesic curvature be the same at any given point for all the curves through that point.

Such families are characterized by equations of the form $\gamma_{0}=G(x, y)$, $x$ and $y$ being any curvilinear coördinates upon the surface. The most interesting particular case occurs when $G(x, y)$ is identically zero, which condition defines the geodesics of the surface. The geodesics of any surface have therefore the property $V(a, b) \equiv V(b, a)$. This is the content (for $n=2$ ) of the theorem of Levi-Civita enunciated at the beginning of this paper. LeviCivita's result thus enters as a special case under the theorem stated above.

\section{THEORY FOR ORDINARY THREE-DIMENSIONAL SPACE}

10. Generalities; definitions. In extending the preceding considerations to space, we have to consider a family of curves defined by two differential equations of the second order:

$$
\begin{aligned}
& Y^{\prime \prime}=F\left(X, Y, Z, Y^{\prime}, Z^{\prime}\right) \\
& Z^{\prime \prime}=G\left(X, Y, Z, Y^{\prime}, Z^{\prime}\right) .
\end{aligned}
$$

Such a family consists of $\infty^{4}$ curves, one for each initial point and direction

- The geometric properties of curve systems of the type (15) have been studied by $\mathrm{K}$. Ogurs, On the integral curves of ordinary differential equations of the second order of a certain type, Tohoku Mathematical Journal, vol. 8 (1915), pp. 93-107. 
$x, y, z, p, q$. Let the equations of the curve corresponding to these initial values be

$$
\begin{aligned}
& Y=Y(X ; x, y, z, p, q) \\
& Z=Z(X ; x, y, z, p, q),
\end{aligned}
$$

so that (2) represents the general solution of (1). We can write the equations (2) explicitly as power series; the first terms are

$$
\begin{aligned}
& Y=y+p(X-x)+\frac{1}{2} F(x, y, z, p, q)(X-x)^{2}+\cdots \\
& Z=z+q(X-x)+\frac{1}{2} G(x, y, z, p, q)(X-x)^{2}+\cdots
\end{aligned}
$$

If we select any two points of space $a$ and $b$, there will be, under the proper restrictions, ${ }^{*}$ a unique curve $C$ of the family (1) passing through these points. Let an element of area $\delta \sigma_{1}$ be situated at $b$ normal to $C$; the curves that join the boundary of $\delta \sigma_{1}$ to $a$ form at $a$ a curvilinear cone of infinitesimal solid angle $\delta \omega_{1}$. Then we define:

$$
V(a, b)=\frac{\delta \sigma_{1}}{\delta \omega_{1}}
$$

A similar definition determines $V(b, a)$, and as in the theory for the plane we introduce also the function

$$
W(a, b)=\frac{V(a, b)}{V(b, a)} .
$$

In order to derive formulas for $V$ and $W$ we refer the neighborhood of $a$ to a rectangular axis system $\xi, \eta, \zeta$ of which the $\zeta$-axis is the tangent to $C$ at $a$. Similarly, the neighborhood of $b$ is referred to an axis system $u, v, w$ where $w$ is the tangent to $C$ at $b . \dagger$ We designate the plane $\xi, \eta$ as $\pi_{1}$, the plane $u$, $v$ as $\pi_{2}$.

The curves of the given family as they stream away from $a$ and impinge upon the plane $\pi_{2}$ set up a correspondence between the directions through $a$ and the points of $\pi_{2},-a$ correspondence which for directions infinitely near to $\zeta$ and therefore for points infinitely near to $b$ is linear (affine). Now $V(a, b)$ is equal to the determinant of this linear correspondence. For $V(a, b)$ may be interpreted as the ratio of two areas correlated with one another affinely, and under an affine transformation all areas are multiplied by the determinant.

11. The functions $\eta(X), \zeta(X)$ and their linear differential equations. Let the Cartesian coördinates of $a$ and $b$ be respectively $\left(x_{1}, y_{1}, z_{1}\right)$, $\left(x_{2}, y_{2}, z_{2}\right)$. Suppose the equations of $C$ to be

$$
Y=y(X), \quad Z=z(X) ;
$$

* See Picard, Traite d'Analyse, vol. 3, pp. 90-99.

In the choice of each axis system there is, of course, one degree of freedom, but this circumstance is indifferent for what follows. 
then the direction of $C$ at $a$ is defined by the parameters

$$
p_{1}=y^{\prime}\left(x_{1}\right), \quad q_{1}=z^{\prime}\left(x_{1}\right),
$$

and at $b$ by the parameters

$$
p_{2}=y^{\prime}\left(x_{2}\right), \quad q_{2}=z^{\prime}\left(x_{2}\right) .
$$

The equations (3) of $C$ will result upon the substitution of $x_{1}, y_{1}, z_{1}, p_{1}, q_{1}$ for $x, y, z, p, q$ in the equations (2); also upon the substitution of $x_{2}, y_{2}, z_{2}$, $p_{2}, q_{2}$ for the same letters.

Let $C_{1}$ be a curve, of the family under consideration, passing through $a$ in a direction $p_{1}+\delta p_{1}, q_{1}+\delta q_{1}$ infinitely near to that of $C$. The equations of $C_{1}$ result by writing in (2):

$$
x=x_{1}, \quad y=y_{1}, \quad z=z_{1}, \quad p=p_{1}+\delta p_{1}, \quad q=q_{1}+\delta q_{1} .
$$

When we then expand according to powers of $\delta p_{1}, \delta q_{1}$ we may omit the powers beyond the first as negligible in the limit, and find for the equations of $C_{1}$ :

$$
\begin{aligned}
& Y=y(X)+\delta p_{1} \eta_{1}(X)+\delta q_{1} \eta_{2}(X) \\
& Z=z(X)+\delta p_{1} \zeta_{1}(X)+\delta q_{1} \zeta_{2}(X),
\end{aligned}
$$

where we have written

$$
\begin{aligned}
& \eta_{1}(X)=Y_{p}\left(X ; x_{1}, y_{1}, z_{1}, p_{1}, q_{1}\right) \\
& \zeta_{1}(X)=Z_{p}\left(X ; x_{1}, y_{1}, z_{1}, p_{1}, q_{1}\right) \\
& \eta_{2}(X)=Y_{q}\left(X ; x_{1}, y_{1}, z_{1}, p_{1}, q_{1}\right) \\
& \zeta_{2}(X)=Z_{q}\left(X ; x_{1}, y_{1}, z_{1}, p_{1}, q_{1}\right) .
\end{aligned}
$$

In the same way the equations of the curve $C_{2}$ passing through $b$ in a direction $p_{2}+\delta p_{2}, q_{2}+\delta q_{2}$ will be

$$
\begin{aligned}
& Y=y(X)+\delta p_{2} \eta_{3}(X)+\delta q_{2} \eta_{4}(X) \\
& Z=z(X)+\delta p_{2} \zeta_{3}(X)+\delta q_{2} \zeta_{4}(X)
\end{aligned}
$$

where system of two linear differential equations of the second order.* For since (2) represents the solution of the equations (1), there will result upon substitution

*The equations of variation of the differential equations (1).

Trans. Am. Math. Soc. 20 
of (2) in (1) two identities in $X, x, y, z, p, q$. Differentiating these identities with respect to $p$, we obtain:

$$
\begin{aligned}
& \left(Y^{\prime \prime}\right)_{p}=F_{y} Y_{p}+F_{z} Z_{p}+F_{y^{\prime}}\left(Y^{\prime}\right)_{p}+F_{z^{\prime}}\left(Z^{\prime}\right)_{p} \\
& \left(Z^{\prime \prime}\right)_{p}=G_{y} Y_{p}+G_{z} Z_{p}+G_{y^{\prime}}\left(Z^{\prime}\right)_{p}+G_{z^{\prime}}\left(Z^{\prime}\right)_{p} .^{*}
\end{aligned}
$$

Assuming the derivatives involved to be continuous, the differentiations with respect to $X$ and $p$ are commutative, and the above equations may be rewritten:

$$
\begin{aligned}
& \left(Y_{p}\right)^{\prime \prime}=F_{y}\left(Y_{p}\right)+F_{z}\left(Z_{p}\right)+F_{y^{\prime}}\left(Y_{p}\right)^{\prime}+F_{z^{\prime}}\left(Z_{p}\right)^{\prime} \\
& \left(Z_{p}\right)^{\prime \prime}=G_{y}\left(Y_{p}\right)+G_{z}\left(Z_{p}\right)+G_{y^{\prime}}\left(Y_{p}\right)^{\prime}+G_{z^{\prime}}\left(Z_{p}\right)^{\prime} .
\end{aligned}
$$

For $x, y, z, p, q$ equal to $x_{1}, y_{1}, z_{1}, p_{1}, q_{1}, Y_{p}, Z_{p}$ reduce to $\eta_{1}(X), \zeta_{1}(X)$. It follows that $\eta_{1}, \zeta_{1}$ obey the linear differential equations

$$
\begin{aligned}
& \eta^{\prime \prime}(X)=F_{y^{\prime}} \eta^{\prime}(X)+F_{z^{\prime}} \zeta^{\prime}(X)+F_{y} \eta(X)+F_{z} \zeta(X) \\
& \zeta^{\prime \prime}(X)=G_{y^{\prime}} \eta^{\prime}(X)+G_{z^{\prime}} \zeta^{\prime}(X)+G_{y} \eta(X)+G_{z} \zeta(X),
\end{aligned}
$$

where it is understood that, in the coefficients $F_{y^{\prime}}\left(X, Y, Z, Y^{\prime}, Z^{\prime}\right)$, etc., the substitutions

$$
Y=y(X), \quad Z=z(X) . \quad Y^{\prime}=y^{\prime}(X), \quad Z^{\prime}=z^{\prime}(X)
$$

are to be made, so that these coefficients are functions only of $X$, the abscissa along $C$.

In similar manner the other systems $\eta, \zeta$ can be shown to obey the equations (5). Each $\eta, \zeta$ is therefore determined if initial values are given for $\eta, \zeta$, $\eta^{\prime}, \zeta^{\prime}$. For $\eta_{1}, \zeta_{1}$ such values are found by differentiating $\left(2^{\prime}\right)$ with respect to $p$, and then replacing $x, y, z, p, q$ by $x_{1}, y_{1}, z_{1}, p_{1}, q_{1}$; this gives:

$$
\left(6_{1}\right) \quad \eta_{1}\left(x_{1}\right)=0, \quad \zeta_{1}\left(x_{1}\right)=0, \quad \eta_{1}^{\prime}\left(x_{1}\right)=1, \quad \zeta_{1}^{\prime}\left(x_{1}\right)=0 \text {. }
$$

$$
\begin{array}{ll}
\eta_{1}(X)=\left(X-x_{1}\right)+\frac{1}{2} F_{p}\left(x_{1}, y_{1}, z_{1}, p_{1}, q_{1}\right)\left(X-x_{1}\right)^{2}+\cdots \\
\zeta_{1}(X)= & \frac{1}{2} G_{p}\left(x_{1}, y_{1}, z_{1}, p_{1}, q_{1} ;\right)\left(X-x_{1}\right)^{2}+\cdots ;
\end{array}
$$

Similarly we find

$$
\begin{aligned}
& \left(6_{3}\right) \quad \eta_{3}\left(x_{2}\right)=0, \quad \zeta_{3}\left(x_{2}\right)=0, \quad \eta_{3}^{\prime}\left(x_{2}\right)=1, \quad \zeta_{3}^{\prime}\left(x_{2}\right)=0 \text {, } \\
& \text { (64) } \quad \eta_{4}\left(x_{2}\right)=0, \quad \zeta_{4}\left(x_{2}\right)=0, \quad \eta_{4}^{\prime}\left(x_{2}\right)=0, \quad \zeta_{4}^{\prime}\left(x_{2}\right)=1 \text {. }
\end{aligned}
$$

The fact important for the present developments is that the four systems $\eta, \zeta$, as a fundamental system of independent solutions of the equations (5),

* For convenience of notation, small letters are used instead of capitals in denoting the partial derivatives of the functions $F$ and $G$. 
must obey the relation

$$
\left|\begin{array}{llll}
\eta_{1}(X) & \eta_{2}(X) & \eta_{3}(X) & \eta_{4}(X) \\
\zeta_{1}(X) & \zeta_{2}(X) & \zeta_{3}(X) & \zeta_{4}(X) \\
\eta_{1}^{\prime}(X) & \eta_{2}^{\prime}(X) & \eta_{3}^{\prime}(X) & \eta_{4}^{\prime}(X) \\
\zeta_{1}^{\prime}(X) & \zeta_{2}^{\prime}(X) & \zeta_{3}^{\prime}(X) & \zeta_{4}^{\prime}(X)
\end{array}\right|=e^{\int_{k}^{X}\left(F_{y^{\prime}}+G_{z^{\prime}}\right) d X},
$$

where $k$ is an undetermined constant. In (7), put $X=x_{1}$; then by reference to $\left(6_{1}\right),\left(6_{2}\right)$,

$$
\left|\begin{array}{ll}
\eta_{3}\left(x_{1}\right) & \eta_{4}\left(x_{1}\right) \\
\zeta_{8}\left(x_{1}\right) & \zeta_{4}\left(x_{1}\right)
\end{array}\right|=e^{\int_{k}^{x_{1}}\left(F_{y^{\prime}}+G_{8^{\prime}}\right) d x}
$$

Similarly, by putting $X=x_{2}$ and referring to $\left(6_{3}\right),\left(6_{4}\right)$, we have

$$
\left|\begin{array}{ll}
\eta_{1}\left(x_{2}\right) & \eta_{2}\left(x_{2}\right) \\
\zeta_{1}\left(x_{2}\right) & \zeta_{2}\left(x_{2}\right)
\end{array}\right|=e^{\int_{k}^{s_{3}}\left(F_{y^{\prime}}+G_{z^{\prime}}\right) d x} .
$$

On dividing $\left(8_{1}\right)$ into $\left(8_{2}\right), k$ is eliminated; we find

$$
\frac{\left|\begin{array}{ll}
\eta_{1}\left(x_{2}\right) & \eta_{2}\left(x_{2}\right) \\
\zeta_{1}\left(x_{2}\right) & \zeta_{2}\left(x_{2}\right)
\end{array}\right|}{\left|\begin{array}{ll}
\eta_{3}\left(x_{1}\right) & \eta_{4}\left(x_{1}\right) \\
\zeta_{3}\left(x_{1}\right) & \zeta_{4}\left(x_{1}\right)
\end{array}\right|}=e^{\int_{x_{1}}^{x_{2}}\left(F_{y^{\prime}}+G_{\prime^{\prime}}\right) d x} .
$$

This result will be directly useful in deriving an expression for $W(a, b)$.

12. Expression for $V(a, b)$. Recurring to $\$ 10$, we next deduce an expression for the linear currespondence there described.

The curve $C_{1}$ which passes through $a$ in the direction $p_{1}+\delta p_{1}, q_{1}+\delta q_{1}$ will pierce the plane $\pi_{2}$ in a point $b_{1}$ of coördinates $x_{2}+\delta x_{2}, y_{2}+\delta y_{2}, z_{2}+\delta z_{2}$. These coördinates must satisfy the equations $\left(3_{1}\right)$ of $C_{1}$; therefore

$$
\begin{aligned}
y_{2}+\delta y_{2} & =y\left(x_{2}+\delta x_{2}\right)+\delta p_{1} \eta_{1}\left(x_{2}+\delta x_{2}\right)+\delta q_{1} \eta_{2}\left(x_{2}+\delta x_{2}\right), \\
z_{2}+\delta z_{2} & =z\left(x_{2}+\delta x_{2}\right)+\delta p_{1} \zeta_{1}\left(x_{2}+\delta x_{2}\right)+\delta q_{1} \zeta_{2}\left(x_{2}+\delta x_{2}\right),
\end{aligned}
$$

whence, omitting infinitesimals of higher order than the first,

$$
\begin{aligned}
\delta y_{2} & =p_{2} \delta x_{2}+\delta p_{1} \eta_{1}\left(x_{2}\right)+\delta q_{1} \eta_{2}\left(x_{2}\right) \\
\delta z_{2} & =q_{2} \delta x_{2}+\delta p_{1} \zeta_{1}\left(x_{2}\right)+\delta q_{1} \zeta_{2}\left(x_{2}\right) .
\end{aligned}
$$

Let the direction cosines of the $u, v$ axes at $b$ be

those of the $w$-axis are

$$
\begin{aligned}
& u: a, b, c \\
& v: l, m, n ;
\end{aligned}
$$

$$
w: \frac{1}{\sqrt{1+p_{2}^{2}+q_{2}^{2}}}, \frac{p_{2}}{\sqrt{1+p_{2}^{2}+q_{2}^{2}}}, \frac{q_{2}}{\sqrt{1+p_{2}^{2}+q_{2}^{2}}} .
$$


Since $u, v, w$ are a trirectangular system, we have the relations

$$
\begin{aligned}
a+p_{2} b+q_{2} c & =0 \\
l+p_{2} m+q_{2} n & =0 \\
b n-c m & =\frac{1}{\sqrt{1+p_{2}^{g}+q_{2}^{2}}} .
\end{aligned}
$$

At the same time we may turn our attention to the $\xi, \eta, \zeta$ system at $a$, where, writing for the direction cosines

$$
\begin{gathered}
\text { of } \xi: \alpha, \beta, \gamma, \\
\text { of } \eta: \lambda, \mu, \nu, \\
\text { of } \zeta: \frac{1}{\sqrt{1+p_{1}^{2}+q_{1}^{2}}}, \frac{p_{1}}{\sqrt{1+p_{1}^{2}+q_{1}^{2}}}, \frac{q_{1}}{\sqrt{1+p_{1}^{2}+q_{1}^{2}}},
\end{gathered}
$$

we note the relations

$$
\begin{aligned}
\alpha+p_{1} \beta+q_{1} \gamma & =0, \\
\lambda+p_{1} \mu+q_{1} \nu & =0, \\
\beta \nu-\gamma \mu & =\frac{1}{\sqrt{1+p_{1}^{2}+q_{1}^{2}}} .
\end{aligned}
$$

The coördinates $u, v$ of $b_{1}$ in the plane $\pi_{2}$ are connected with the space coördinates $\delta x_{2}, \delta y_{2}, \delta z_{2}$ as follows:

Hence by (10),

$$
\begin{aligned}
& u=a \delta x_{2}+b \delta y_{2}+c \delta z_{2} \\
& v=l \delta x_{2}+m \delta y_{2}+n \delta z_{2} .
\end{aligned}
$$

$$
\begin{array}{r}
u=\left(a+p_{2} b+q_{2} c\right) \delta x_{2}+\left[b \eta_{1}\left(x_{2}\right)+c \zeta_{1}\left(x_{2}\right)\right] \delta p_{1}+\left[b \eta_{2}\left(x_{2}\right)+c \zeta_{2}\left(x_{2}\right)\right] \delta q_{1} \\
v=\left(l+p_{2} m+q_{2} n\right) \delta x_{2}+\left[m \eta_{1}\left(x_{2}\right)+n \zeta_{1}\left(x_{2}\right)\right] \delta p_{1} \\
+\left[m \eta_{2}\left(x_{2}\right)+n \zeta_{2}\left(x_{2}\right)\right] \delta q_{1}
\end{array}
$$

but by (11) the coefficients of $\delta x_{2}$ are zero, so that the equations connecting $u, v$ with $\delta p_{1}, \delta q_{1}$ are

$$
\begin{aligned}
u & =\left[b \eta_{1}\left(x_{2}\right)+c \zeta_{1}\left(x_{2}\right)\right] \delta p_{1}+\left[b \eta_{2}\left(x_{2}\right)+c \zeta_{2}\left(x_{2}\right)\right] \delta q_{1} \\
v & =\left[m \eta_{1}\left(x_{2}\right)+n \zeta_{1}\left(x_{2}\right)\right] \delta p_{1}+\left[m \eta_{2}\left(x_{2}\right)+n \zeta_{2}\left(x_{2}\right)\right] \delta q_{1} .
\end{aligned}
$$

For temporary expediency we call the coefficients in (13) $A, B, C, D$, writing these equations as

$$
\begin{aligned}
u & =A \delta p_{1}+B \delta q_{1} \\
v & =C \delta p_{1}+D \delta q_{1} .
\end{aligned}
$$


The next step is to pass from $\delta p_{1}, \delta q_{1}$ as coördinates for directions through $a$ to $\xi, \eta$. $\xi$ is the cosine of the angle between $1: p_{1}+\delta p_{1}: q_{1}+\delta q_{1}$ and $\alpha, \beta, \gamma$, hence

$$
\xi=\frac{\alpha+\beta\left(p_{1}+\delta p_{1}\right)+\gamma\left(q_{1}+\delta q_{1}\right)}{\sqrt{1+p_{1}^{2}+q_{1}^{2}}}=\frac{\beta \delta p_{1}+\gamma \delta q_{1}}{\sqrt{1+p_{1}^{2}+q_{1}^{2}}},
$$

on account of the first equation (12). In the same way,

$$
\eta=\frac{\lambda+\mu\left(p_{1}+\delta p_{1}\right)+\nu\left(q_{1}+\delta q_{1}\right)}{\sqrt{1+p_{1}^{2}+q_{1}^{2}}}=\frac{\mu \delta p_{1}+\nu \delta q_{1}}{\sqrt{1^{*}+p_{1}^{2}+q_{1}^{2}}},
$$

ky the second of the equations (12).

Solving for $\delta p_{1}, \delta q_{1}$, we have

$$
\begin{aligned}
& \delta p_{1}=\left(1+p_{1}^{2}+q_{1}^{2}\right)(\nu \xi-\gamma \eta), \\
& \delta q_{1}=\left(1+p_{1}^{2}+q_{1}^{2}\right)(-\mu \xi+\beta \eta),
\end{aligned}
$$

wherein we have used the third equation (12).

Carrying these values for $\delta p_{1}, \delta q_{1}$ into $\left(13^{\prime}\right)$, there is obtained for the linear correspondence between $\xi, \eta$ and $u, v$ :

$$
\begin{aligned}
& u=\left(1+p_{1}^{2}+q_{1}^{2}\right)\{(\nu A-\mu B) \xi-(\gamma A-\beta B) \eta\}, \\
& v=\left(1+p_{1}^{2}+q_{1}^{2}\right)\{(\nu C-\mu D) \xi-(\gamma C-\beta D) \eta\} .
\end{aligned}
$$

We have said that $V(a, b)$ is the determinant of this correspondence. So

$$
V(a, b)=\left(1+p_{1}^{2}+q_{1}^{2}\right)^{2}\left|\begin{array}{ll}
\nu & \mu \\
\gamma & \beta
\end{array}\right| \cdot\left|\begin{array}{ll}
A & B \\
C & D
\end{array}\right| .
$$

Introducing the expansions of $A, B, C, D$ as in (13), we obtain

$$
V(a, b)=\left(1+p_{1}^{2}+q_{1}^{2}\right)^{2}\left|\begin{array}{ll}
\nu & \mu \\
\gamma & \beta
\end{array}\right| \cdot\left|\begin{array}{ll}
b & c \\
m & n
\end{array}\right| \cdot\left|\begin{array}{ll}
\eta_{1}\left(x_{2}\right) & \eta_{2}\left(x_{2}\right) \\
\zeta_{1}\left(x_{2}\right) & \zeta_{2}\left(x_{2}\right)
\end{array}\right|,
$$

and then, by reference to (11) and (12),

$$
V(a, b)=\frac{\left(1+p_{1}^{2}+q_{1}^{2}\right)^{3 / 2}}{\left(1+p_{2}^{2}+q_{2}^{2}\right)^{1 / 2}}\left|\begin{array}{ll}
\eta_{1}\left(x_{2}\right) & \eta_{2}\left(x_{2}\right) \\
\zeta_{1}\left(x_{2}\right) & \zeta_{2}\left(x_{2}\right)
\end{array}\right| .
$$

From $\left(15_{1}\right) V(b, a)$ is at once deducible by the systematic interchange of $a$ and $b$ :

$$
V(b, a)=\frac{\left(1+p_{2}^{2}+q_{2}^{2}\right)^{8 / 2}}{\left(1+p_{1}^{2}+q_{1}^{2}\right)^{1 / 2}}\left|\begin{array}{ll}
\eta_{8}\left(x_{1}\right) & \eta_{4}\left(x_{1}\right) \\
\zeta_{8}\left(x_{1}\right) & \zeta_{4}\left(x_{1}\right)
\end{array}\right| .
$$

13. Expression for $W(a, b)$. The preceding formulas show that the determination of $V(a, b)$ requires in general the solution of a system of two linear differential equations of the second order. But, as in the two-dimensional 
theory, it appears that $W(a, b)$, on the contrary, can be expressed by a quadrature.

For, by division of $\left(15_{1}\right)$ by $\left(15_{2}\right)$,

$$
W(a, b)=\frac{V(a, b)}{V(b, a)}=\frac{\left(1+p_{1}^{2}+q_{1}^{2}\right)^{2}}{\left(1+p_{2}^{2}+q_{2}^{2}\right)^{2}} \frac{\left|\begin{array}{ll}
\eta_{1}\left(x_{2}\right) & \eta_{2}\left(x_{2}\right) \\
\zeta_{1}\left(x_{2}\right) & \zeta_{2}\left(x_{2}\right)
\end{array}\right|}{\left|\begin{array}{ll}
\eta_{3}\left(x_{1}\right) & \eta_{4}\left(x_{1}\right) \\
\zeta_{3}\left(x_{1}\right) & \zeta_{4}\left(x_{1}\right)
\end{array}\right|}
$$

and it has been shown at the end of $\S 11$ that

Therefore

$$
\frac{\left|\begin{array}{ll}
\eta_{1}\left(x_{2}\right) & \eta_{2}\left(x_{2}\right) \\
\zeta_{1}\left(x_{2}\right) & \zeta_{2}\left(x_{2}\right)
\end{array}\right|}{\left|\begin{array}{ll}
\eta_{3}\left(x_{1}\right) & \eta_{4}\left(x_{1}\right) \\
\zeta_{3}\left(x_{1}\right) & \zeta_{4}\left(x_{1}\right)
\end{array}\right|}=e^{\int_{x_{1}}^{x_{2}}\left(F_{y^{\prime}}+G_{z^{\prime}}\right) d x}
$$

$$
W(a, b)=\frac{\left(1+p_{1}^{2}+q_{1}^{2}\right)^{2}}{\left(1+p_{2}^{2}+q_{2}^{2}\right)^{2}} \int^{\int_{x_{1}}^{x_{2}}\left(F_{y^{\prime}}+G_{z^{\prime}}\right) d x} .
$$

An equivalent form is:

$$
W(a, b)=e^{\int_{x_{1}}^{x_{2}}\left(F_{y^{\prime}}+G_{z^{\prime}}-4 \frac{y^{\prime} F+z G^{\prime}}{1+y^{\prime 2}+z^{\prime 2}}\right) d x} .
$$

14. The invariance of $\left(\partial \gamma_{1} / \partial \theta_{1}\right)+\left(\partial \gamma_{2} / \partial \theta_{2}\right)$. It is desirable to obtain an expression of $W(a, b)$ in geometrically intrinsic terms. For this, we must find a quantity to play the rôle of the $\partial \gamma / \partial \theta$ of the two-dimensional theory. Such a quantity is furnished by the following considerations.

Given any family of $\infty^{4}$ curves in space,

$$
\begin{aligned}
& y^{\prime \prime}=F\left(x, y, z, y^{\prime}, z^{\prime}\right) \\
& z^{\prime \prime}=G\left(x, y, z, y^{\prime}, z^{\prime}\right),
\end{aligned}
$$

each point and direction (lineal element) define a curve. Let $O, O t$ be any point and direction of space, determining the curve $C$.

Construct a rectangular axis system $O t, O u, O v$ of which $O t$ is one of the axes. In this, it is to be emphasized, there is one degree of arbitrariness, for the axis system may be rotated freely about $O t$.

The $u$ and $t$ axes define a pencil of directions, and accordingly a pencil of curves. These form a surface $S_{1}$ tangent to the plane $u t$. In the same way the $v$ axis defines with the $t$ axis a pencil of curves, which form a surface $S_{2}$ tangent to the plane $v t$.

The surfaces $S_{1}$ and $S_{2}$ intersect in the curve $C$. Introducing here the idea of geodesic curvature, we direct attention to the fact that the geodesic curvature of a curve upon a surface is relative to both surface and curve. Let the geodesic curvature at $O$ of $C^{\prime}$ be $\gamma_{1}$ upon $S_{1}$, and $\gamma_{2}$ upon $S_{2}$. 
Regard $S_{1}$. It is covered with a pencil of curves issuing from 0 . Let $\partial \gamma_{1} / \partial \theta_{1}$ denote the rate at which the geodesic curvature changes in this pencil as we pass from $C$ to the infinitesimally adjacent curves. And let $\partial \gamma_{2} / \partial \theta_{2}$ denote the corresponding quantity for the surface $S_{2}$.

Then we state the

ThEOREM. The sum $\left(\partial \gamma_{1} / \partial \theta_{1}\right)+\left(\partial \gamma_{2} / \partial \theta_{2}\right)$ is independent of the one degree of freedom involved in the choice of the trirectangular system $t, u, v$.

To prove this, suppose the curve family referred to $t, u, v$ as $x, y, z$ axes; then, taking advantage of the fact that geodesic curvature is equal to tangential curvature, we have

$$
\frac{\partial \gamma_{1}}{\partial \theta_{1}}=F_{y^{\prime}}, \quad \frac{\partial \gamma_{2}}{\partial \theta_{2}}=G_{z^{\prime}} .
$$

If the $t, u, v$ system is rotated about $t$ through an angle $\varphi$, we find that, relative to the new trirectangular system,

$$
\begin{aligned}
& \frac{\partial \gamma_{1}}{\partial \theta_{1}}=\cos ^{2} \varphi F_{y^{\prime}}+\sin \varphi \cos \varphi\left(F_{z^{\prime}}+G_{y^{\prime}}\right)+\sin ^{2} \phi G_{z^{\prime}}, \\
& \frac{\partial \gamma_{2}}{\partial \theta_{2}}=\sin ^{2} \varphi F_{y^{\prime}}-\sin \varphi \cos \varphi\left(F_{z^{\prime}}+G_{y^{\prime}}\right)+\cos ^{2} \varphi G_{z^{\prime}} .
\end{aligned}
$$

Adding, the angle $\varphi$ disappears:

$$
\frac{\partial \gamma_{1}}{\partial \theta_{1}}+\frac{\partial \gamma_{2}}{\partial \theta_{2}}=F_{y^{\prime}}+G_{z^{\prime}},
$$

whence the invariantive property of $\left(\partial \gamma_{1} / \partial \theta_{1}\right)+\left(\partial \gamma_{2} / \partial \theta_{2}\right)$ as stated.

With this result established, the formula (16) can be given the form:

$$
W(a, b)=\frac{V(a, b)}{V(b, a)}=e^{\int_{a}^{b}\left(\frac{\partial \gamma_{1}}{\partial \theta_{1}}+\frac{\partial \gamma_{2}}{\partial \theta_{2}}\right) d s} .
$$

15. The case of symmetric $V(a, b)$. The families of $\infty^{4}$ curves in space for which $V(a, b)$ is symmetric in its two arguments are found immediately from considering the formulas (16), (17).

TheOREM. The curve families for which

are those for which

$$
V(a, b) \equiv V(b, a)
$$

$$
\frac{\partial \gamma_{1}}{\partial \theta_{1}}+\frac{\partial \gamma_{2}}{\partial \theta_{2}} \equiv 0 .^{*}
$$

* A more graphical form of this condition is as follows: We can construct an indicatrix to show the variation of curvature; namely, $O, O t$ being any point and direction, by laying off on every ray $O u$ perpendicular to $O t$ a length proportional to $(\partial \gamma / \partial \theta)^{-1 / 2}$ relative to the pencil defined by $O u, O t$. The indicatrix is in every case a conic with $O$ as center. When $V(a, b)$ is symmetric every indicatrix is an equilateral hyperbola, and conversely. 
How is the property $V(a, b) \equiv V(b, a)$ to be recognized in a given family of $\infty^{4}$ curves from the analytic form of the differential equations? According to (16) the necessary and sufficient condition for the property in question is

$$
F \cdot y^{\prime}+G_{z^{\prime}}-4 \frac{y^{\prime} F+z^{\prime} G}{1+y^{\prime 2}+z^{\prime 2}}=0 \text {. }
$$

This equation is transformable into

$$
\frac{\partial}{\partial y^{\prime}}\left[\frac{F}{\left(1+{y^{\prime 2}}^{2}+{z^{\prime 2}}^{2}\right)^{2}}\right]+\frac{\partial}{\partial z^{\prime}}\left[\frac{G}{\left(1+{\left.y^{\prime 2}+z^{\prime 2}\right)^{2}}^{\prime}\right.}\right]=0 .
$$

Hence the result:

The families of $\infty^{4}$ curves that have the property $V(a, b) \equiv V(b, a)$ are characterized by differential equations of the form

$$
\begin{aligned}
& y^{\prime \prime}=\left(1+{y^{\prime}}^{2}+{z^{\prime}}^{2}\right)^{2} \Omega_{1}, \\
& z^{\prime \prime}=\left(1+y^{\prime 2}+{z^{\prime}}^{2}\right)^{2} \Omega_{2},
\end{aligned}
$$

where $\Omega_{1}, \Omega_{2}$ are any two functions of $x, y, z, y^{\prime}, z^{\prime}$ that obey the relation

$$
\frac{\partial \Omega_{1}}{\partial y^{\prime}}+\frac{\partial \Omega_{2}}{\partial z^{\prime}}=0 \text {. }
$$

The general solution of (18) is

$$
\begin{aligned}
& \Omega_{1}=\frac{\partial}{\partial z^{\prime}} \phi\left(x, y, z, y^{\prime}, z^{\prime}\right), \\
& \Omega_{2}=-\frac{\partial}{\partial y^{\prime}} \phi\left(x, y ; z, y^{\prime}, z^{\prime}\right),
\end{aligned}
$$

where $\phi$ is any function of its five arguments. The type of curve system here under consideration depends therefore on one arbitrary function of five arguments, whereas the general system of $\infty^{4}$ curves in space depends on two such functions.

16. Straubel's theorem; its converse. Straubel's theorem for three-dimensional space results from applying the formula (16) for $W(a, b)$ to the case of a natural family of curves. Such a family, consisting of the paths of light in an isotropic optical medium, is determined as the system of extremals of $\int \nu(x, y, z) d s, \nu$ being the index of refraction,- or by the equivalent differential equations

$$
\begin{aligned}
& y^{\prime \prime}=\left(L_{y}-y^{\prime} L_{x}\right)\left(1+{y^{\prime}}^{2}+{z^{\prime}}^{2}\right), \\
& z^{\prime \prime}=\left(L_{z}-z^{\prime} L_{x}\right)\left(1+{y^{\prime}}^{2}+{z^{\prime}}^{2}\right),
\end{aligned}
$$

where $L=\log \nu .^{*}$

* Cf. E. Kasner, loc. cit. 
Applying (16) to the equations (19), we find

$$
\frac{V(a, b)}{V(b, a)}=\frac{\nu^{2}(a)}{\nu^{2}(b)} \text {. }
$$

Consider the converse question. In connection with what systems of $\infty^{4}$ curves can a function of position $\nu$ be defined so that the relation (20) shall subsist? By use of (16), it turns out that this is possible whenever the differential equations are of the form

$$
\begin{aligned}
& y^{\prime \prime}=\left(L_{y}-y^{\prime} L_{x}\right)\left(1+{y^{\prime}}^{2}+{z^{\prime}}^{2}\right)+\left(1+{y^{\prime}}^{2}+{z^{\prime}}^{2}\right)^{2} \Omega_{1}, \\
& z^{\prime \prime}=\left(L_{z}-z^{\prime} L_{x}\right)\left(1+{y^{\prime}}^{2}+{z^{\prime}}^{2}\right)+\left(1+{y^{\prime 2}}^{\prime 2}{z^{\prime 2}}^{2}\right)^{2},
\end{aligned}
$$

where $\Omega_{1}, \Omega_{2}$ are any two functions of $x, y, z, y^{\prime}, z^{\prime}$ subject to the condition

$$
\frac{\partial \Omega_{\mathrm{i}}}{\partial y^{\prime}}+\frac{\partial \Omega_{2}}{\partial z^{\prime}}=0 \text {. }
$$

The Straubel property is therefore not characteristic of natural families.

17. Flat (Euclidean) $n$-dimensional space. The preceding theorems admit of easy generalization to a flat $n$-space. Using as notation for the coördinates $x, y_{1}, y_{2}, \cdots, y_{n-1}$, a system of $n-1$ differential equations of the second order,

$$
y_{i}^{\prime \prime}=F_{i}\left(x, y_{j} ; y_{j}^{\prime}\right) \quad(i, j=1,2, \cdots, n-1)
$$

will define a system of $\infty^{2 n-2}$ curves. Such a system will have the property $V(a, b) \equiv V(b, a)$ when the differential equations are of the form

$$
y_{i}^{\prime \prime}=\left(1+y_{1}^{\prime 2}+\cdots+y_{n-1}^{\prime 2}\right)^{(n+1) / 2} \Omega_{i} \quad(i=1,2, \cdots, n-1),
$$

where the $\Omega$ 's are any functions of the $x, y_{j}, y_{j}^{\prime}$ that obey the relation

$$
\cdot \frac{\partial \Omega_{1}}{\partial y_{1}^{\prime}}+\frac{\partial \Omega_{2}}{\partial y_{2}^{\prime}}+\cdots+\frac{\partial \Omega_{n-1}}{\partial y_{n-1}^{\prime}}=0 .
$$

The Straubel property, which for $n$-space is

$$
\frac{V(a, b)}{V(b, a)}=\frac{\nu^{n-1}(a)}{\nu^{n-1}(b)}
$$

is found to be characteristic of the families defined by differential equations of the form:

$$
\begin{aligned}
y_{i}^{\prime \prime}=\left(L_{y_{i}}-y_{i}^{\prime} L_{x}\right)\left(1+y_{1}^{\prime 2}+\cdots\right. & \left.+y_{n-1}^{\prime 2}\right) \\
& +\left(1+y_{1}^{\prime 2}+\cdots+y_{n-1}^{\prime 2}\right)^{(n+1) / 2} \Omega_{i} \\
& (i=1,2, \cdots, n-1),
\end{aligned}
$$

where the $\Omega$ 's are subject to the condition (24). 
We note that the general solution of (24) is obtained by taking any $(n-2)$ functions

$$
\phi_{1}, \phi_{2}, \cdots, \phi_{n-2}
$$

of the arguments $x, y_{j}, y_{j}^{\prime}$ and setting the $\Omega$ 's equal to the jacobians:*

$$
\Omega_{1}=\frac{\partial\left(\phi_{1}, \phi_{2}, \cdots, \phi_{n-2}\right)}{\partial\left(y_{2}^{\prime}, y_{3}^{\prime}, \cdots, y_{n-1}^{\prime}\right)}, \quad \Omega_{2}^{\prime}=-\frac{\partial\left(\phi_{1}, \phi_{2}, \cdots, \phi_{n-2}\right)}{\partial\left(y_{1}^{\prime}, y_{3}^{\prime}, \cdots, y_{n-1}^{\prime}\right)}, \quad \text { etc. }
$$

The families with symmetric $V(a, b)$ depend therefore on one less arbitrary function of the $2 n-1$ arguments $x, y_{j}, y_{j}^{\prime}$ than the general family of $\infty^{2 n-2}$ curves.

\section{Theory for a general (CURVEd, NON-EUclidean) VARIETy OF $n$ DIMENSIONS}

18. The invariance of $\Sigma\left(\partial \gamma_{i} / \partial \theta_{i}\right)$. Formula for $W(a, b)$. We extend our developments, finally, to a general variety $V_{n}$, one in which the squared element of length is defined by the general quadratic form,

$$
d s^{2}=\sum a_{i k} d x_{i} d x_{k} .
$$

The first step is to generalize the theorem of part II concerning the invariance of $\left(\partial \gamma_{1} / \partial \theta_{1}\right)+\left(\partial \gamma_{2} / \partial \theta_{2}\right)$. We are considering a system of $\infty^{2 n-2}$ curves in $V_{n}$, one for each point and direction. Let the point $O$ and direction $O t$ define the curve $C$. Then let a system of $n$ rectangular directions be constructed of which $O t$ is one: $O t, O t_{1}, \cdots, O t_{n-1}$. Each $O t_{i}$ determines with $O t$ a pencil of directions, therefore a pencil of curves, which form a two-spread $V_{2}^{(i)}$. There arise in this way $n-1 V_{2}$ 's, all intersecting in the curve $C$. On each $V_{2}^{(i)}, C$ has at $O$ a definite geodesic curvature $\gamma_{i}$, which changes at a definite rate $\partial \gamma_{i} / \partial \theta_{i}$ as we pass from $C$ to the infinitesimally adjacent curves upon $V_{2}^{(i)}$.

Now, in the construction of the rectangular system $\mathrm{Ot}_{i}$ it is seen that there exist $\frac{1}{2}(n-1)(n-2)$ degrees of freedom. Hence the significance of the

Theorem. The sum

$$
\sum_{i=1}^{i=n-1} \frac{\partial \gamma_{i}}{\partial \theta_{i}}
$$

is independent of the $\frac{1}{2}(n-1)(n-2)$ degrees of freedom involved in the construction of the rectangular system $O t, O t_{1}, \cdots, O t_{n-1}$.

Upon this result we can then establish the theorem:

$$
W(a, b)=e^{\int_{a}^{b} \sum_{i=1}^{n-1} \frac{\partial \gamma_{i}}{\partial \theta_{i}} d s}
$$

From the form of the last equation there follow for a general space the same

- See De La Vallee Poussin, Cours d'Analyse, vol. 2, p. 302. 
corollaries as in $\S \S 4,5$ of the theory for the plane. It follows also that the necessary and sufficient condition for

is

$$
V(a, b) \equiv V(b, a)
$$

$$
\sum_{i=1}^{i=n-1} \frac{\partial \gamma_{i}}{\partial \theta_{i}} \equiv 0 \text {. }
$$

19. Geodesics. Levi-Civita's theorem. Consider the most important example of $\infty^{2 n-2}$ curves in $V_{n}$-the geodesics. A geodesic in $V_{n}$ is a fortiori a geodesic upon any sub-vaviety $V_{m}$ upon which it may lie. Recalling the meaning of $\gamma_{i}$ as defined in the preceding section, we see that for the geodesics we have, separately and identically,

$$
\gamma_{1}=0, \quad \gamma_{2}=0, \quad \cdots, \quad \gamma_{n-1}=0,
$$

and hence, separately and identically,

$$
\frac{\partial \gamma_{1}}{\partial \theta_{1}}=0, \quad \frac{\partial \gamma_{2}}{\partial \theta_{2}}=0, \quad \cdots, \quad \frac{\partial \gamma_{n-1}}{\partial \theta_{n-1}}=0 .
$$

From these equations there follows, according to (2),

Levi-Civita's Theorem.* The geodesics of any $n$-dimensional variety have the property $V(a, b) \equiv V(b, a)$.

Levi-Civita's result thus falls as a special case under the theorem stated above in connection with the equation (2).

20. Conformal representation. Straubel's theorem and its generalization. In conclusion, we consider Straubel's theorem for a general variety $V_{n}$. Taking the Straubel relation

$$
\frac{V(a, b)}{V(b, a)}=\frac{\nu^{n-1}(a)}{\nu^{n-1}(b)},
$$

we inquire as to the most general family of $\infty^{2 n-2}$ curves in $V_{n}$ in connection with which a point-function $\nu$ can be defined so that the stated relation shall hold.

To this end, conformal representation furnishes the easiest method. Let the manifold $V_{n}$, of which the fundamental form is

$$
d s^{2}=\sum a_{i k} d x_{i} d x_{k},
$$

be represented upon a second manifold $V_{n}^{\prime}$ of which the fundamental form is

$$
d s_{1}^{2}=\nu^{2} \sum a_{i k} d x_{i} d x_{k}
$$

so that points with the same coördinates correspond. Since $d s_{1}=\nu d s$, the mapping so defined is conformal with modulus of conformality $\nu$.

* See the introduction. 
The behavior of $V(a, b)$ under the conformal transformation is seen to be as follows:

By division,

$$
\begin{aligned}
& V\left(a_{1}, b_{1}\right)=\nu^{n-1}(b) V(a, b), \\
& V\left(b_{1}, a_{1}\right)=\nu^{n-1}(a) V(b, a) .
\end{aligned}
$$

$$
\frac{V\left(a_{1}, b_{1}\right)}{V\left(b_{1}, a_{1}\right)}=\frac{\nu^{n-1}(b)}{\nu^{n-1}(a)} \frac{V(a, b)}{V(b, a)} .
$$

Hence if in $V_{n}$ we have the condition (3), then in $V_{n}^{\prime}$ we shall have

$$
V\left(a_{1}, b_{1}\right)=V\left(b_{1}, a_{1}\right),
$$

and conversely.

The relation (7) holds in particular for the geodesics of $V_{n}^{\prime}$ (Levi-Civita's theorem). These are the extremals of

$$
\int d s_{1}=\min . ;
$$

the image curves in $V_{n}$ are therefore the extremals of

$$
\int \nu d s=\min . ;
$$

they are, in other words, a natural family. Thus all natural families have the Straubel property.

But the property (7) belongs not only to the geodesics of $V_{n}^{\prime},-i t$ holds for every system of $\infty^{2 n-2}$ curves that satisfy the condition $\Sigma\left(\partial \gamma_{i} / \partial \theta_{i}\right) \equiv 0$. Hence the Straubel property is not restricted to natural families, that is to the conformal images of geodesics, but is true of the more inclusive type that results by conformal mapping from any family with the symmetric property $V(a, b) \equiv V(b, a)$, or its equivalent $\Sigma\left(\partial \gamma_{i} / \partial \theta_{i}\right) \equiv 0$.

Colombia UNIVERSITY 